\title{
Sugar and Organic Acid Changes in Stored Onion Bulbs Treated with Maleic Hydrazide
}

\author{
A.M. Salamal, J.R. Hicks ${ }^{2}$, and J.F. Nock ${ }^{3}$ \\ Vegetable Crops Department, Cornell University, Ithaca, NY 14853
}

Additional index words. Allium cepa, soluble sugars, organic acids

\begin{abstract}
Maleic hydrazide (MH)-treated and untreated (control) onion (Allium cepa L.) bulbs were stored for up to 20 weeks at 0,15 , or $30 \mathrm{C}$ with relative humidities (RH) of $40 \%$ or $60 \%$. MH and RH had minimal effect on sugars and organic acids in inner or outer scale leaves that were analyzed at $\mathbf{S}$-week intervals. Concentrations of fructose, glucose, and total sugars were higher in inner than outer leaves of the bulb, while the reverse was true for sucrose. Total sugars, glucose, and fructose decreased and sucrose increased with higher storage temperature. Total sugars and glucose decreased with increased storage duration. Malic acid concentration was greater in the outer leaves while citric acid levels were higher in inner leaves. Malic acid increased in onion bulbs during storage while citric acid levels were not influenced by storage duration. Total acids showed little difference across temperatures, due to the concurrent increase in citric acid and decrease in malic acid at 30C.
\end{abstract}

The commercial storage period for onions is often terminated by the initiation of root growth and sprout elongation. Immediately after harvest, the bulbs are in a natural state of rest that is controlled by endogenous hormone levels and varies with genetic makeup of the particular cultivar. Within a given cultivar, regrowth may be delayed or controlled by use of maleic hydrazide (MH) (Isenberg and Ang, 1963), low (0C) or high temperature (30C+) (Abdalla and Mann, 1963; Karamkar and Joshi, 1941) and, to a much lesser extent, by low humidity (Ogata, 1961; van den Berg and Lentz, 1973). The effect of $\mathrm{MH}$ is to inhibit mitosis in the meristematic regions (Greulach and Atchison, 1950; Masters et al., 1984; Pendergrass, 1969) while low temperature acts by slowing all metabolic reaction within the bulb. The high temperature effect, although well-documented and widely used in some regions, is not wellunderstood. Humidity levels, if controlled at all, are usually manipulated to reach a balance between decay development, moisture loss, skin color, and regrowth.

Adequate metabolic substrate levels (primarily carbohydrate but may also include organic acid) are essential for storage of any crop. In onions, fructose level al harvest has been suggested as an indicator of storage potential (Rutherford and Whittle, 1982, 1984). Gorin and Borcsok (1980) considered total sugar content during storage to be an index of keeping quality. However, Pendergrass (1969) found that MH prevented regrowth at 4C but had no effect on either carbohydrate

Received for publication 25 Aug. 1989. The cost of publishing this paper was defrayed in part by the payment of page charges. Under postal regulations, this paper therefore must be hereby marked advertisement solely to indicate this fact.

${ }^{1}$ Former Graduate Student.

${ }^{2}$ Associate Professor.

${ }^{3}$ Research Technician. or organic acid levels. In potatoes and cabbage there have been differences reported in levels of malic and/or citric acids in response to storage temperature and duration (Hicks and Ludford, 1980; Hicks et al., 1982: Shekhar and Iritani, 1979).

Carbohydrates in onion bulbs account for a major portion of their dry weight and include fructose, glucose, sucrose, a series of oligosaccharides, and possibly arabinose and ribose (Bacon, 1957; Pendergrass, 1969: Rutherford and Whittle, 1982). Organic acids that have been identified include pyruvic, malic, citric, fumaric, and $\alpha-$ ketoglutaric (Bennett, 1945; Pendergrass, 1969; Take and Otsuka, 1967).

In our work some of the major metabolic substrates (glucose, fructose, sucrose, malic acid, citric acid) were derermined under a series of storage conditions that inhibit or promote regrowth. Since there are conflicting reports on the influence of sugars on the storage life of onion bulbs, our objective was to separate the effects of storage conditions from those of regrowth on changes in individual sugars. In addition, since there are reported differences in the major organic acids in onions, changes that probably are due to melabolic utilization during storage, these too were investigated. The effects of storage conditions and $\mathrm{MH}$ treatment on sugars and organic acids are also discussed in relation to quality changes reported previously (Salama and Hicks, 1987).

Experiments were conducted using 'Sentinel' onion bulbs commercially grown on oreanic soils in New York state. Half of the onions received the MH treatment. The diethanolamine salt of $\mathrm{MH}$ (1,2-dihydro-3,6pyridazinedione) was applied at the rate of $2.24 \mathrm{~kg} \cdot \mathrm{ha}^{-1} 2$ weeks before harvest. At harvest, bulbs were hand-pulled and field-cured for 1 week. The tops were manually removed, and the bulbs cured in crates at $\approx 25 \mathrm{C}$, $60 \%$ to $70 \% \mathrm{RH}$ for an additional 2 weeks.
Table 1. Sugar levels (mg.g ${ }^{-1}$ dry weight) in the inner and outer leaves of onion bulbs.

\begin{tabular}{lcccc}
\hline \hline \multirow{2}{*}{$\begin{array}{lcc}\text { Scaf } \\
\text { location }\end{array}$} & \multicolumn{4}{c}{ Sugar $^{2}\left(\mathrm{mg} \cdot \mathrm{g}^{-1}\right)$} \\
\cline { 2 - 5 } & Fruciose & Glucosc & Sucrosc & Total \\
\hline Inncr & 108 & 209 & 151 & 467 \\
Outer & 129 & 231 & 158 & 518 \\
Significancc & $\cdots$ & $\cdots$ & $\cdots$ & $\cdots$ \\
\hline
\end{tabular}

'Mean of 144 values (four storage times $\times$ three temperatures $\times$ two $\mathrm{RH} \times$ two $\mathrm{MH}$ treatments $\mathrm{X}$ three replicates).

**,***F tests were significant at $P=0.01$ and 0.001 , respectively.

Table 2. Sucrose levels (mg.g ${ }^{-1}$ dry weight) in inner and outer leaves at three storage temperatures.

\begin{tabular}{lccc}
\hline \hline & \multicolumn{3}{c}{ Sucrose $\left(\mathrm{mg} \cdot \mathrm{g}^{-1}\right)$} \\
\cline { 2 - 4 } Leaf & \multicolumn{3}{c}{ Temperaturc $\left({ }^{\circ} \mathrm{C}\right)^{2}$} \\
\cline { 2 - 4 } location & 0 & 15 & 30 \\
\hline Inncr & 135 & 140 & 177 \\
Outcr & 128 & 149 & 196 \\
Significance & NS & - & $\cdots$ \\
\hline
\end{tabular}

${ }^{\mathrm{z}}$ Mean of 48 values (four storage times $\times$ two RH $x$ two MH treatments $x$ three replicates).

$\mathrm{NS},{ }^{*}, * * \mathrm{~F}$ tests were nonsignificant or significant at $P=0.05$ or 0.01 , respectively.

Table 3. Organic acid levels ( $\mathrm{mg} \cdot \mathrm{g}^{-1}$ dry weight) in the inner and outer leaves of onion bulbs.

\begin{tabular}{lccc}
\hline \hline \multirow{2}{*}{$\begin{array}{l}\text { Ocaf } \\
\text { location }\end{array}$} & \multicolumn{3}{c}{ Organic acid $\left(\mathrm{mg} \cdot \mathrm{g}^{-1}\right)$} \\
\cline { 2 - 4 } & Malate & Citratc & Total \\
\hline Inncr & 26 & 23 & 49 \\
Outer & 33 & 13 & 46 \\
Significance & $\cdots$ & $\cdots$ & $*$ \\
\hline
\end{tabular}

${ }^{\mathrm{z}}$ Mean of 144 values (four storage rimes $\mathrm{x}$ three temperatures $\times$ two $\mathrm{RH} \times$ two $\mathrm{MH}$ treatments $x$ three replicates).

$*, * * * \mathrm{~F}$ tests were significant at $P=0.05$ and 0.01 , respectively.

Table 4. Malate levels (mg. $\mathrm{g}^{-1}$ dry weight) in inner and outer leaves at three storage temperatures.

\begin{tabular}{lccc}
\hline \hline & \multicolumn{3}{c}{ Malate $\left(\mathrm{mg} \cdot g^{-1}\right)$} \\
\cline { 2 - 4 } Leaf & \multicolumn{3}{c}{ Temperature $\left({ }^{\circ} \mathrm{C}\right)^{2}$} \\
\cline { 2 - 4 } leation & 0 & 15 & 30 \\
\hline Inner & 27 & 35 & 16 \\
Outer & 40 & 37 & 20 \\
Significance & $\cdots$ & NS & NS \\
\hline
\end{tabular}

${ }^{\mathrm{z}}$ Mean of 48 values (four storage times $\times$ two $\mathrm{RH}$ $x$ two MH treatments $x$ three replicates).

NS,$* * * \mathrm{~F}$ tests were nonsignificant or significant at $\mathrm{P}=0.001$, respectively.

Bulbs were stored in 19-liter plastic containers connected to a flow-through air sysrem. Storage temperatures were 0,15 , and $30 \mathrm{C}$ with $40 \%$ and $60 \% \mathrm{RH}$. The incoming air was passed through glycerol-water solutions to obtain the desired humidities (Johnson, 1940). The experiment was set up in a randomized complete-block design in a factorial arrangement with the following main effects: two MH treatments $(+,-)$. two storage $\mathrm{RH}$, two bulb sampling locations, three storage temperatures, four removal times, and three replicates.

At 5-week intervals, 11 to 14 bulbs with 

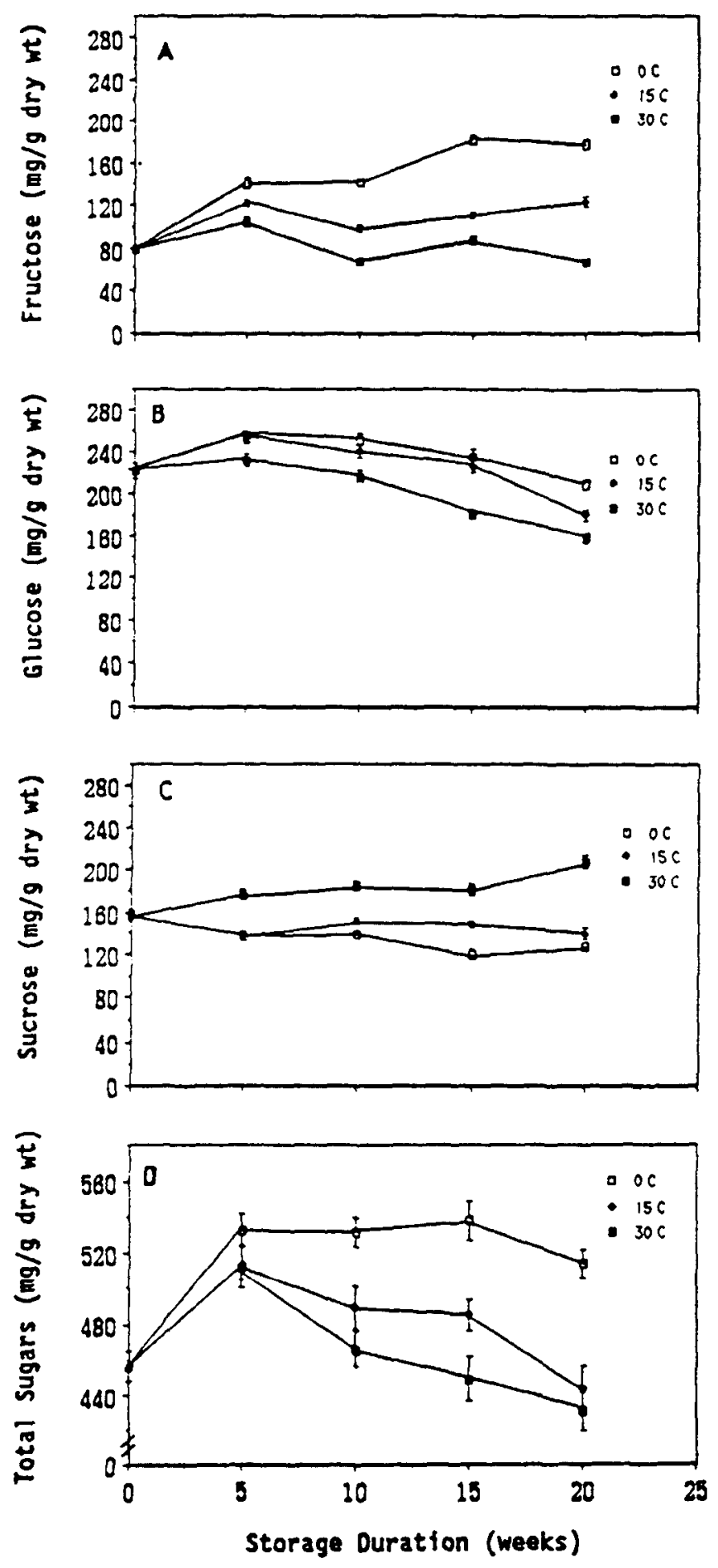

Fig. 1. Effect of storage duration at 0, 15, and 30C on the concentration of glucose (A), fructose (B). sucrose (C) and total sugars (D) in onion bulbs. Vertical lines represent SE.

a total weight of 0.7 to $0.9 \mathrm{~kg}$ were removed for analysis. After the dry outer scale leaves were removed, the first five leaves constituted the outer sample and the remaining part the inner sample. Samples were frozen, freeze-dried, and ground to $0.4 \mathrm{~mm}$ particle size for sugar and organic acid analysis.

Sugars were extracted from $0.5 \mathrm{~g}$ of onion powder in a $50 \mathrm{C}$ water bath with $20 \mathrm{ml}$ of $80 \%$ ethanol containing $2 \mathrm{ml}$ of $2.5 \%$ (phenyl$\beta-D-$ glucoside) as an internal standard. After centrifugation, the supernatant was decanted and the pellet re-extracted twice with $20 \mathrm{~m}$ ! solution) was added as an internal standard to $0.25 \mathrm{~g}$ of onion powder and the acids were extracted twice with $20 \mathrm{ml} 80 \%$ ethanol in a $50 \mathrm{C}$ water bath. After centrifugation, the supernatants were combined and the organic acids precipitated with lead acetate. The precipitate was washed with $80 \%$ ethanol and anhydrous ethyl ether, air-dried, and then oven-dried at $45 \mathrm{C}$ overnight. Trimethylsilyl derivatives were made from the dried powder in a manner similar to that used for the carbohydrate derivatives.

For both sugars and organic acids, $2 \mu \mathrm{l}$ of the trimethylsilyl derivatives was injected into a Hewlett Packard Model 5710A gas chromatograph equipped with a flame ionization detector and connected to a Hewlett Packard Model 3390A integrator. A stainless steel column ( $3 \mathrm{~m} \times 2 \mathrm{~mm}$ i.d.) packed with $3 \%$ OV-101 on 80/100 mesh Supelcoport was used. The column temperature for the sugars was held for $2 \mathrm{~min}$ at $190 \mathrm{C}$ and programmed to $270 \mathrm{C}$ at the rate of $8 \mathrm{C} / \mathrm{min}$. For organic acids, the column temperature was held at $135 \mathrm{C}$ for $2 \mathrm{~min}$ and then programmed to increase at $8 \mathrm{C} / \mathrm{min}$ to $240 \mathrm{C}$. The injector and detector were at 300 and $350 \mathrm{C}$, respectively. Nitrogen was used as the carrier gas at a flow rate of $30 \mathrm{ml} \cdot \mathrm{min}^{-1}$. Retention times and response factors for sugars and acids were verified daily by injection of standard solutions.

Data were tested by analysis of variance using the general linear models procedures of SAS (1985). Significant interactions were further analyzed using single-degree-of-freedom contrasts. Sugar and organic acid levels before storage are reported but not included in the analysis since they did not fit into the factorial design.

The sugars detected were fructose, a- and P-glucose, and sucrose. The RH level had no significant effect on sugar changes during storage. The main effects and interaction of temperature and storage duration were significant for individual and total sugars.

Fructose concentration increased during storage at 0 and $15 \mathrm{C}$ (Fig. I), as noted by Rutherford and Whittle (1982, 1984) for onions stored at 0C. At 30C fructose decreased to one-third and one-half that of onions stored 20 weeks at 0 or $15 \mathrm{C}$, respectively. Since the major storage polysaccharides in onion bulbs are fructans (Bacon, 1959; Darbyshire and Henry, 1978), which are composed almost entirely of fructose units, fructose would be expected as the primary product of hydrolysis.

Glucose content increased slightly during the first $\mathrm{S}$ weeks at 0 or $15 \mathrm{C}$, and then gradually decreased at all temperatures (Fig. I), with the decrease being greater at higher temperatures. At $0 \mathrm{C}$ the magnitude of the decrease in glucose was similar to that of the increase in fructose.

Sucrose content decreased slightly after 5 weeks in storage at 0 or $15 \mathrm{C}$ but increased slightly during that same period at $30 \mathrm{C}$. Sucrose showed an additional increase by 20 weeks of storage at $30 \mathrm{C}$, which implies a net synthesis.

Total sugars (fructose + glucose + su- 

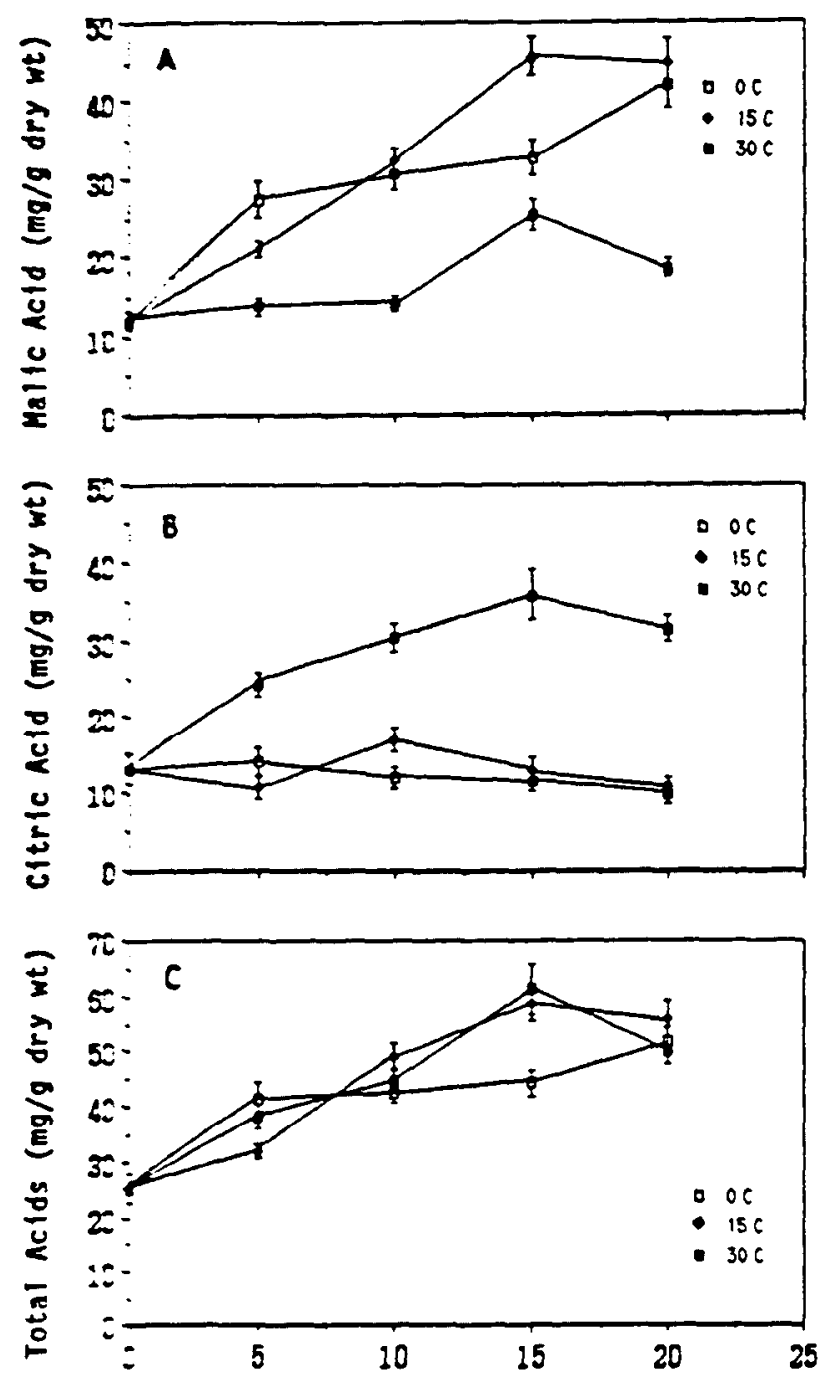

\section{Storage Duration (weeks)}

Fig. 2. Effect of storage duration at 0,15 , and $30 \mathrm{C}$ on the concentration of malic (A), citric (B), and total acids in onion bulbs. Vertical lines represent SE.

crose) decreased the 5th week, and this decrease was greater at $30 \mathrm{C}$ than at $15 \mathrm{C}$ (Fig. 1). Changes at $0 \mathrm{C}$ were small. Fructose increased over time at this temperature, balancing the drop in glucose during longer storage periods. The decrease in total sugars at 15 and $30 \mathrm{C}$ was primarily due to the decrease in glucose and probably reflects increased sugar utilization in metabolic activities at higher temperature

Fructose and glucose were higher in the outer than in the inner leaves (Table 1). This was also true for sucrose, with the exception of the $0 \mathrm{C}$ treatment where there was no difference (Table 2). Ogata 1961) reported lower sugar levels in outer leaves while Rutherford and Whittle (1982) found no major difference in sugar content of inner and outer scales.

Gorin and Borcsok's 1980 suggestion that maximum sugar content could he an indicator of "freshness" in onion was based on their report of an increase in sucrose and fructose during 5 months of storage of untreated bulbs, with no increase MH-treated bulbs. Our data show no influence of a pre- harvest MH spray on fructose, glucose, or sucrose levels during storage. Regrowth (total number of bulbs showing external sprouts or growth of new roots) during the 20 weeks of storage ranged from a low of $3.3 \%$ at $0 \mathrm{C}$ up to $4.7 \%$ at $15 \mathrm{C}$ for $\mathrm{MH}$-treated and $11.8 \%$. $13.8 \%$, and $25.3 \%$ for untreated bulbs at 30 , 0 , and $15 \mathrm{C}$, respectively. The majority of the regrowth was due to rooting and occurred during the latter part of the storage period. Sprout growth may create a greater demand for mobilization and utilization of carbohydrates than does root growth; this possibility would explain why our results differ from those of Rutherford and Whittle (1982) who found that when fructose in onion bulbs continued to increase in storage at $4 \mathrm{C}$, sprouting was delayed in comparison with when fructose content stopped increasing earlier. However, bulbs that showed root growth in our tests likely also had initiated shoot growth that had not yet reached the surface of the bulbs.

Malic and citric, the major organic acids in onion bulbs, comprised $2.8 \%$ and $1.8 \%$ of the dry weight, respectively. Fumaric and succinic were present, but in very low amounts. The main effects of temperature and storage time were significant as was the interaction of these factors for each of the two predominant acids and total acids (malic + citric).

Higher amounts of malic acid were found after storage at 0 and 15 than at $30 \mathrm{C}$ (Fig. 2 ), which agrees with findings of Pendergrass (1969), confirming that malic acid is the predominant organic acid in onion bulbs stored at low temperatures. Increased concentrations of malic acid have also been observed in low temperature storage of potatoes (Shekhar and Iritani, 1979) and cabbage (Hicks and Ludford, 1980: Hicks et al., 1982). Rate of metabolic activity could not be the only explanation since slightly more malic acid accumulated in bulbs at 15 than at $0 \mathrm{C}$ while the respiration rate at $15 \mathrm{C}$ is more than twice that at 0C (Salama and Hicks, 1987).

Malic acid increased with storage duration until 15 weeks, although onions stored at $30 \mathrm{C}$ only exhibited this increase at 15 weeks (Fig. 2). At 20 weeks, onions stored at 0C continued to show an increase in malic acid while those stored at $30 \mathrm{C}$ showed a decline.

Citric acid generally exhibited less fluctuation than malic acid over time at 0 and $15 \mathrm{C}$ and was similar to initial values (Fig. 2 ), whereas it increased at $30 \mathrm{C}$. The ratio of citrate : malate varied from approximately $1: 5$ at 0 and $15 \mathrm{C}$ to $2: 1$ at $30 \mathrm{C}$. These data suggest that some of the conflicting reports on whether the major organic acid in onion bulbs is malic (Pendergrass, 1969) or citric (Take and Orsuka, 1967) may be related to storage temperature and duration.

Differences in total acids due to temperature were minimized primarily by the higher citric acid and lower malic acid content in onions held a: 30C (Fig. 2). MH and RH levels had no significant effect on organic acid changes in storage.

The amount of malic acid was higher in outer leaves than inner leaves (Table 3 ) only with onions stored at 0C (Table 4). The amount of citric acid was higher in inner leaves than outer leaves (Table 3).

Temperature was the major factor influencing the sugar and acid changes in onion bulbs during storage with the most dramatic differences due to storage at 30C. The large change in the citric : malic ratio shows a major impact on metabolic processes. In addition to the exponential effect of temperature on metabolism, Ladeinde and Hicks (1988) have shown that there is a substantial decrease in internal oxygen of storage type onion bulbs at $30 \mathrm{C}$ with the corresponding increase in $\mathrm{CO}_{2}$. In addition to affecting TCA cycle acids, a modified atmosphere around the growing point may play a role in prevention of sprouting a: high temperatures.

\section{Literature Cited}

Abdalla, A.A. and L.K. Mann. 1963. Bulb development in the onion (Allium cepa L.) and the effect of storage temperature on bulb rest. Hilgardia 35(5):85-112.

Bacon. J.S.D. 1957. The water-soluble carbohy- 